\title{
ANÁLISIS COMPARATIVO DE PREDICTORES POTENCIALES DE PRÁCTICAS DISCIPLINARIAS SEVERAS CON PREESCOLARES, ANTES Y DESPUÉS DE UN ENTRENAMIENTO PARA PADRES
}

\author{
Pedro Solís-Cámara*1, Yolanda Medina Cuevas ${ }^{1}$, Marysela Díaz Romero ${ }^{2}$ \\ ${ }^{1}$ Universidad de Guadalajara, ${ }^{2}$ Universidad del Valle de México
}

Recibido, noviembre 4/2013

Concepto de evaluación, septiembre 26/2014

Aceptado, marzo 10/2015

\begin{abstract}
Referencia: Solís-Cámara, P., Medina Cuevas, Y. \& Díaz Romero, M.(2015). Análisis comparativo de predictores potenciales de prácticas disciplinarias severas con preescolares, antes y después de un entrenamiento para padres. Acta Colombiana de Psicología, 18(2), 139-150. DOI: 10.14718/ ACP.2015.18.2.12
\end{abstract}

Resumen

\begin{abstract}
El objetivo de este estudio consistió en determinar si un entrenamiento en pautas de crianza dirigido a padres con niños de tres a cinco años de edad modificaría las prácticas disciplinarias severas, así como algunos predictores potenciales de esas prácticas, como son el coraje-agresión y el estrés de los padres, al igual que el estrés en la interacción entre padres e hijos, y los problemas de conducta y el estrés de los niños. Sesenta padres (41 mamás y 19 papás) contestaron los cuestionarios correspondientes. La muestra se dividió al azar en grupo experimental (GE: expuesto a un entrenamiento en crianza) y grupo control (GC: sin entrenamiento). Los padres contestaron los mismos cuestionarios diez semanas después. Un análisis de regresión jerárquica $(\mathrm{ARJ})$ con la muestra total mostró que el estrés en la interacción y las conductas problema fueron las principales variables predictoras de las prácticas disciplinarias $(p<.01)$. En la segunda evaluación, los resultados indicaron la modificación significativa de las prácticas disciplinarias y de todas las variables del estudio en el GE, pero no en el GC; además, nuevos ARJ con el GC y con el GE indicaron la importancia del coraje-agresión parental como variable predictora de las prácticas disciplinarias. Los resultados se discuten en términos de la importancia de la modificación de las prácticas disciplinarias severas y sus efectos negativos en los niños, así como del uso adecuado de técnicas cognitivo-conductuales para modificar positivamente las relaciones negativas entre los padres y el niño.

Palabras clave: coraje-agresión, disciplina, entrenamiento, padres, preescolares, problemas de conducta.
\end{abstract}

\section{COMPARATIVE ANALYSIS OF PREDICTIVE FACTORS OF SEVERE DISCIPLINARY PRACTICES WITH PRESCHOOLERS, BEFORE AND AFTER PARENT TRAINING}

\begin{abstract}
The objective of this study consisted on determining whether training parents of preschool children on rearing practices would modify harsh disciplinary practices as well as some potential predictors of these practices, such as anger-aggression and stress of parents, children's stress and behavioral problems, as well as parent-child stress. Sixty parents (41 moms and 19 dads) answered the corresponding questionnaires. The sample was randomly divided into an experimental group (EG), exposed to parent training, and a control group without training (CG). Parents answered the same questionnaires 10 weeks later. Hierarchical regression analysis (HRA) showed that parent-child stress and behavioral problems were the main predictor variables of disciplinary practices $(p<.01)$ in the total sample. In the second assessment, results indicated significant changes in disciplinary practices and all the variables for the EG, but not for the CG. In addition, HRA performed on the CG and the EG pointed out the importance of parental anger-aggression as a variable predictive of disciplinary practices. Results are discussed in terms of the importance of modifying harsh disciplinary practices and their negative effects on children, as well as the proper use of cognitive-behavioral techniques to modify negative relationships between parents and children.

Key words: anger-aggression, behavior problems, discipline practices, parent training, young children.
\end{abstract}

\footnotetext{
* Laboratorio de Psicología: Investigación e Intervención del Centro de Investigación en Conducta y Cognición Comparada. CUCiénega. Universidad de Guadalajara. Av. Universidad, Núm.1115, Col. Lindavista, Ocotlán, Jalisco. México. Código Postal 47820. Teléfono: (+52+392) 925 9400, ext. 48496. Correo electrónico: psolis@mexis.com. Los autores agradecen a Diana Cortés, Daniel Iñiguez, Carlos Arambula, Martha Aguirre, Arantxa Martínez, Ricardo Vargas-Zúñiga y Josué Castolo, por su apoyo operativo para el estudio, $\mathrm{y}$, a todos los directivos y personal de estancias infantiles, particulares y oficiales, por su apoyo. Datos parciales de este estudio fueron presentados en el VII Congreso Iberoamericano de Psicología en Oviedo, España, 2010. El estudio fue posible gracias al apoyo otorgado por PROMEP-SEP y la Universidad de Guadalajara.
} 


\title{
ANÁLISE COMPARATIVA DE PREDITORES POTENCIAIS DE PRÁTICAS DISCIPLINARES SEVERAS COM PRÉ-ESCOLARES, ANTES E DEPOIS DE UM TREINAMENTO PARA PAIS
}

\begin{abstract}
Resumo
O objetivo deste estudo consistiu em determinar se um treinamento em pautas de criação dirigido a pais com crianças de três a cinco anos de idade modificaria as práticas disciplinares severas, bem como alguns preditores potenciais dessas práticas, como por exemplo a coragem-agressão e o estresse dos pais, da mesma forma que o estresse na interação entre pais e filhos, e os problemas de conduta e o estresse das crianças. Sessenta pais (41 mães e 19 pais) responderam os questionários correspondentes. A mostra foi dividida aleatoriamente em grupo experimental (GE: exposto a um treinamento em criação) e o grupo de controle (GC: sem treinamento). Os pais responderam os mesmos questionários dez semanas depois. Uma análise de regressão hierárquica (ARH) com a mostra total assinalou que o estresse na interação e as condutas problema foram as principais variáveis preditoras das práticas disciplinares $(p<.01)$. Na segunda avaliação, os resultados indicaram a modificação significativa das práticas disciplinares e de todas as variáveis do estudo no GE, mas não no GC; além disso, novos ARH com o GC e com o GE indicaram a importância da coragem-agressão parental como variável preditora das práticas disciplinares. Os resultados discutem-se em termos da importância da modificação das práticas disciplinares severas e sus efeitos negativos nas crianças, bem como do uso adequado de técnicas cognitivo-condutuais para modificar positivamente as relações negativas entre os pais e a criança.

Palavras chave: coragem-agressão, disciplina, treinamento, pais, pré-escolares, problemas de conduta.
\end{abstract}

\section{INTRODUCCIÓN}

El presente estudio se enmarca en el modelo de determinantes de la crianza de Belsky (1984; Belsky \& Jaffee, 2006). Este modelo de procesos enfatiza el papel de los factores socio-contextuales como moldeadores de la crianza. Es decir, considera los atributos de los niños, la historia de desarrollo de los padres y sus propias características psicológicas, así como el contexto social más amplio en el que se encuentran inmersos los padres y sus niños. Este modelo ha generado evidencias empíricas de los múltiples determinantes que moderan o amplifican los efectos de las prácticas de crianza de los padres en sus niños; entre ellos se encuentran el nivel socio-económico y educativo de la familia, así como el apoyo social y el estrés de los padres, entre otros (e.j., Belsky, 1990; Hill, 2006; Ragozin, Basham, Crnic, Greenberg \& Robinson, 1982; Solís-Cámara \& Díaz-Romero, 2002).

Una de las prácticas de crianza considerada como un factor de riesgo para la socialización de los niños es el uso del castigo físico como técnica disciplinaria, el cual incluye otros comportamientos como los gritos por parte de los padres, y que en este estudio englobamos con el término de prácticas disciplinarias severas. Se conceptualizan las prácticas disciplinarias de los padres como acciones o comportamientos aprendidos por ellos, ya sea a raíz de su propia experiencia de imitación y que se realizan para guiar las conductas de los niños (e.j., le grito a mi niña(o) por tirar la comida). A pesar de que estas prácticas se consideran como un factor de riesgo para los niños, Berzenski y Yates (2013) argumentan que la demostración de, por ejemplo, problemas de comportamiento en los niños como resultado de tales prácticas ha sido objeto de discusión por décadas (véase: Berzenski \& Yates, 2013). Uno de los puntos medulares de discusión en los estudios sobre el tema es que estos evalúan el efecto de múltiples determinantes o de variables predictoras de manera aislada y no en conjunto (Baumrind, Larzelere \& Cowan, 2002). Por ejemplo, en un análisis meta analítico de 88 estudios, Gershoff (2002) confirma la importancia de las prácticas disciplinarias severas como un factor de riesgo para los niños; sin embargo, la misma autora señala deficiencias metodológicas de los estudios analizados, incluyendo el problema mencionado antes aquí. Por su parte, Baumrind et al (2002) en su revisión del estudio de Gershoff (2002), concluyen que el análisis sobre los mediadores potenciales que afectan las relaciones entre el castigo físico y sus resultados en los niños indica que la mayoría de los estudios a la fecha han sido simplistas. Y agregan que lo que falta preguntar en los estudios es en cuáles contextos, que tipo de método disciplinario tiene mayor probabilidad de incrementar los resultados positivos o negativos en los niños.

Por otra parte, en un estudio en el que participaron varios cientos de padres gemelos (monocigóticos y dicigóticos), Lynch et al. (2006) encontraron, entre otros hallazgos, que cuando las madres o los padres gemelos utilizaban castigo físico severo (golpes y patadas) con sus respectivos hijos, los problemas de conducta externalizadores del niño(a) eran mayores que en las familias que no utilizaban el castigo severo. Pero el resultado que llama más la atención es el de las familias discordantes en la utilización de castigo físico severo; es decir, cuando una madre o padre utilizaba 
el castigo físico severo con su niño(a) y la madre o padre gemelo utilizaba formas benignas de disciplina con sus propios hijos, los problemas de conducta externalizadores, tales como golpear, también fueron mayores en el primer caso que en el segundo. Como señalan Lynch et al. (2006), este tipo de resultados es el que se esperaría si las estrategias disciplinarias severas fuesen realmente una de las causas de los resultados negativos en los niños. Estudios como este son muy relevantes y congruentes con los señalamientos de autores reconocidos en esta área de investigación (véase: Collins, Maccoby, Steinberg, Hetherington \& Bornstein, 2000) sobre la indudable relación entre lo que hacen o dejan de hacer los padres y el resultado en sus niños.

Es por esto que el presente trabajo considera los efectos sumatorios de varios determinantes o predictores potenciales de las prácticas disciplinarias severas (Baumrind et al., 2002). Además, el estudio se diseñó con el propósito de demostrar que la enseñanza de estrategias de crianza positiva favorece la reducción de las prácticas disciplinarias severas y de algunos de sus determinantes.

A continuación se presentan los argumentos que fundamentan la elección de los posibles determinantes de las prácticas disciplinarias severas que se toman en cuenta en este estudio; estos se incluyen como determinantes propios de las características de los niños y de los padres. En cuanto a las primeras, los estudios encontrados señalan que los problemas de conducta de los niños, tanto internalizadores (e.j., problemas emocionales, introversión, somatización y ansiedad) como externalizadores (e.j., problemas de atención y comportamiento, como la agresión), provocan en los padres una serie de dificultades en la crianza, tornándola emocionalmente desapegada y en general problemática; mientras que un niño emocionalmente positivo facilita la crianza y promueve vínculos afectivos seguros (Brunk \& Henggeler, 1984; Goldberg, Clarke-Stewart, Rice \& Dellis, 2002; Pike, McGuire, Hetherington, Reiss \& Plomin, 1996). De acuerdo con Belsky y Jaffee (2006), es indudable que el comportamiento del niño contribuye, junto con otras influencias reconocibles en el contexto, a las variaciones en la crianza de los padres. Por lo tanto, en este estudio se incluyó la valoración de conductas problema del niño.

En cuanto a los padres, sus características psicológicas, por ejemplo coraje y estrés, también ejercen influencia sobre la manera en que manejan a sus niños (Belsky \& Barends, 2002). Los padres predispuestos al coraje (o rabia) tienden a comportarse de forma menos sensible, receptiva y más severa que otros padres de niños preescolares (NICHD Early Child Care Research Network, 1999; Solís-Cámara, Covarrubias, Díaz \& Rivera, 2004), particularmente en situaciones disciplinarias (Clark, Kochanska \& Ready, 2000). Estos comportamientos o formas de practicar la crianza tienen como resultado un incremento en la severidad o intensidad de las conductas problema de los niños. Existen evidencias (Belsky \& Jaffee, 2006; Bugental \& Happaney, 2002; Solís-Cámara et al., 2004) acerca de la influencia de las interacciones recíprocas resultantes de las atribuciones que hacen los padres sobre las causas del comportamiento de los niños (e.j., creer que un niño llora porque quiere molestar a la madre). Este tipo de atribuciones han sido estudiadas en términos del estrés en la crianza (Abidin, 1995; Solís-Cámara et al., 2004).

Además, el estrés de los padres es reconocido ampliamente como un factor adverso a la crianza positiva (Guajardo, Snyder \& Petersen, 2009; McBride, Schoppe \& Rane, 2002; Solís-Cámara et al., 2007). Debido a otras condiciones o circunstancias propias de los padres, como la situación económica, el trabajo y la propia relación de pareja, el estrés puede observarse en algunos casos como un esfuerzo por proteger al niño (Grych, 2002). Sin embargo, en muchos casos puede manifestarse como una crianza severa (Margolin, Oliver \& Medina, 2001) y en una interacción padre-niño poco favorable, expresada incluso como coraje (Conger, Neppl, Kim \& Scaramella, 2003; Lindahl \& Malik, 1999). Por todo esto, en el presente estudio se incluyen tanto medidas de estrés (ítems relacionados con la propia vida personal de los padres y con la interacción padre-niño), como del coraje-agresión de los padres, en el sentido de la expresión abierta de golpes y amenazas.

Finalmente, parece razonable y recomendable que si se logra identificar los determinantes que influyen o favorecen el uso de prácticas disciplinarias severas, se realicen esfuerzos para demostrar su eliminación o, por lo menos, su reducción, aun considerando predisposiciones o características propias de los padres. Por ello se planeó entrenar a los padres de este estudio con un programa de crianza, fundamentado en el hecho, confirmado ampliamente, de que la involucración activa de los padres en clases de crianza donde adquieren habilidades es altamente efectiva para que ellos se relacionen positivamente con el niño (Atkins, Frazier \& Talbott, 2003; Reid, Webster-Stratton \& Beauchaine, 2001; Solís-Cámara \& Díaz-Romero, 2002; Solís-Cámara et al., 2004).

El estudio se diseñó para padres de niños pequeños porque ha sido ampliamente demostrado que las intervenciones tempranas en la vida de los niños tienen mayor probabilidad de cambiar las interacciones poco sensitivas y responsivas, y además los patrones de crianza temprana predicen exitosamente la estabilidad de los mismos por lo menos en los primeros años de vida de los niños (Dallaire \& Weinraub, 2005; Dishion \& Patterson, 1992; Hansen \& Jones, 2010; Lochman \& Wells, 2003; Solís-Cámara et al., 2004). El entrenamiento seleccionado fue un programa de 
crianza estandarizado que ha demostrado cambios positivos en el comportamiento de los niños de padres entrenados (Nicholson, Anderson, Fox \& Brenner, 2002; Solís-Cámara, Fox \& Nicholson, 2000; Weymouth, 2010). El programa tiene un enfoque que lleva a los padres a comprender sus emociones y sustituirlas por pensamientos al relacionarse con sus niños (i.e., de reacciones a respuestas bien pensadas) y de ahí a adquirir conocimientos y habilidades para interactuar con los niños (i.e., técnicas eficientes y comportamientos adecuados); se fundamenta en las estrategias cognitivo-conductuales originales de ese programa (i.e., Alto, Piensa, Pregunta, Responde), que fue diseñado para padres de preescolares (Solís-Cámara, Díaz, Fox \& Fox, 2002).

En resumen, el objetivo de este estudio consistió en conocer si un entrenamiento en crianza para padres con niños de tres a cinco años de edad modificaría las prácticas disciplinarias severas, así como los predictores o determinantes potenciales de esas prácticas. Para lograr el objetivo, se planeó analizar si el comportamiento problema y el estrés de los niños, así como el estrés en la relación padres-niños, el estrés de los padres y su coraje-agresión podrían predecir de manera significativa las prácticas disciplinarias de los padres y, posteriormente, llevar a cabo los entrenamientos y analizar los cambios logrados. El estudio pretende aportar así al conocimiento sobre la modificación de las prácticas disciplinarias y la identificación y modificación de determinantes de las prácticas disciplinarias.

\section{MÉTODO}

Se trata de un estudio con diseño pre experimental con un grupo expuesto al entrenamiento en crianza (ocho semanas) y un grupo control sin intervención.

\section{Participantes}

Se eligió una muestra por conveniencia formada por 60 padres (41 de género femenino y 19 del masculino) de niños preescolares sin diagnóstico de problemas de conducta $(22$ niñas y 38 niños). Las familias fueron contactadas en cuatro estancias infantiles en la ciudad de Guadalajara, México.

Las mamás o los papás de estos niños contestaron los cuestionarios; el promedio de edad de los padres era de 35.3 años $(D T=6.47) ; 86.7 \%$ estaban casados y el resto separados/divorciados; su escolaridad se distribuyó en $36.7 \%$ con estudios básicos, $21.7 \%$ con estudios técnicos (o doce años de estudios), $8.3 \%$ con estudios universitarios incompletos y $33.3 \%$ eran graduados y/o con posgrado; todos los participantes trabajaban y la mayoría de ellos lo hacía como empleados en empresas (61.7\%); $18.3 \%$ practicaban su profesión en forma privada y $20 \%$ eran técnicos/ obreros, o dedicados al comercio menor; en el momento del estudio, 25\% tenían ingresos familiares entre 1 mil y 5 mil pesos (la cantidad mayor era equivalente a $\$ 500$ US en el momento del estudio); el $51.7 \%$ entre 5 mil y 10 mil, y el $23.3 \%$ entre 10 mil y 15 mil. Los hijos de estos padres tenían una media de edad de 3.68 años $(D T=0.79)$.

\section{Instrumentos}

Información de la familia. Cuestionario simple diseñado para el estudio, que solicita información básica sobre la familia, tal como nivel de escolaridad, ocupación e ingresos de los padres.

Escala de Comportamientos para Madres y Padres de Niños Pequeños. La ECMP (Solís-Cámara, 2007; SolísCámara \& Fox, 2007) consta de 99 ítems calificados con una escala Likert de cuatro puntos y distribuidos en tres subescalas: Expectativas del desarrollo, Prácticas de Crianza y Prácticas Disciplinarias. En este estudio sólo se utilizó la subescala de Disciplina que mide las prácticas disciplinarias de los padres ante los comportamientos de los niños, con 31 ítems (e.j., le grito a mi niña(o) por tirar la comida). Los padres califican cada reactivo con una escala de cuatro puntos $(4=$ casi siempre/siempre, $3=$ frecuentemente, 2 $=$ algunas veces, y $1=$ casi nunca/ nunca). Altas puntuaciones indican prácticas disciplinarias severas (e.j., si mi niño(a) llora después de acostarse, le pego en las nalgas) en las que se opta por los gritos y golpes. La consistencia interna promedio utilizando coeficientes alfa es de .90 (Solís-Cámara, Díaz-Romero, et al., 2002).

Cuestionario de Valoración del Comportamiento. El CVC (Behavior Screening Questionnaire) fue desarrollado por Richman y Graham (1971) para la valoración de niños preescolares con problemas de comportamiento y emocionales. Los índices de confiabilidad entre calificadores del CVC van de .77 a .94. Richman, Stevenson y Graham (1975) informaron que había una correlación de .88 entre el CVC y calificaciones clínicas de niños de tres años de edad. Para este estudio se obtuvo la versión utilizada en otros estudios (Solís-Cámara et al., 2004), que valora doce problemas de conducta comunes en niños pequeños (e.j., berrinches, ir al baño, irse a dormir). Los padres calificaron la frecuencia de cada conducta en relación con su niño utilizando una escala de cuatro puntos (de $4=$ casi siempre/siempre hasta $1=$ casi nunca/nunca). Una calificación total para la conducta problema fue computada combinando las calificaciones de los 12 ítems (rango posible $=12$ a 48).

Cuestionario Breve de Coraje y Agresión. El CBCA(Brief Anger Agression Questionnaire, Maiuro, Vitaliano \& Cahn, 1987) es una medida de seis ítems para valorar los niveles de coraje (e.j., a veces siento que me toca la peor parte 
de la vida) y agresión (e.j., me pongo tan enojado(a) que golpeo, aviento, o pateo cosas). Los ítems son calificados en una escala que va de 0 (extremadamente improbable) a 4 (muy probable), con un total de calificaciones de 0 a 24 (la calificación de corte en 9 indica una probabilidad justa de descontrol por coraje o agresión). El CBCA ha demostrado estar correlacionado con una extensa medida de hostilidad; en .78, discrimina exitosamente entre muestras con y sin problemas de coraje y agresión, y es una medida sensitiva al cambio como resultado de programas de intervención (Solís-Cámara et al., 2004).

Índice de Estrés Paterno. El IEP (Parenting Stress Index; Abidin, 1995) es un instrumento de 36 reactivos que miden el estrés en tres áreas: estrés paterno personal (e.j., a menudo siento que no manejo las cosas muybien), estrés relacionado con la interacción del padre y el niño (e.j., algunas veces mi niño hace cosas sólo para molestarme), y el estrés que resulta de las características únicas del niño (e.j., mi niño parecellorar o alterarse más a menudo que la mayoría de los niños). La forma breve del IEP fue lograda de una versión más larga, seleccionando los ítems con la mayor carga factorial en los tres factores mencionados antes. Altas puntuaciones indican mayor estrés. Los ítems se califican en una escala tipo Likert que va de totalmente de acuerdo (5 puntos) hasta totalmente en desacuerdo (1 punto). Las confiabilidades de medidas repetidas y alfa para la calificación total del IEP son de .84 y.91, respectivamente.

\section{Materiales}

Programa de crianza. Se trata de un programa estandarizado, tipo manual, conocido como Programa de Paternidad Estrella PPE (Solís-Cámara, Díaz, Fox \& Fox, 2001). El objetivo del programa consiste en enseñar a los padres de familia los conocimientos, estrategias y técnicas para favorecer una socialización adecuada de sus niños. El programa ofrece un enfoque que lleva a los padres de las emociones al pensamiento (i.e., conocimientos) y de ahí al comportamiento; se fundamenta en las estrategias originales del PPE: Alto, Piensa, Pregunta y Responde. Consta de dos libros de trabajo diseñados específicamente para padres de infantes o preescolares; en este estudio se utilizó el correspondiente a padres de preescolares (Solís-Cámara, Díaz, et al., 2002). El PPE se divide en cuatro segmentos principales y los temas incluyen: etapas del desarrollo, establecimiento de expectativas, técnicas disciplinarias (ignorar, sobrecorrección, consecuencias y tiempo fuera) y técnicas de crianza positiva (cómo dar instrucciones, prestar atención, mirando hacia adelante, actividades recreativas y reforzamiento positivo). El PPE fue enseñado por educadores previamente entrenados.

\section{Protocolo de intervención}

El entrenamiento consiste en cuatro clases de dos horas y media, ofrecidas en el horario de conveniencia de los padres. Los padres reciben un libro de trabajo que corresponde a la edad de sus hijos (infantes o pre-escolares). En la presente investigación se utilizó la versión de preescolares (tres a cinco años), por ser el rango de edad requerido para este estudio.

A continuación se describe el procedimiento utilizado en las sesiones de clase: En la primera clase se introduce a los padres en el programa, incluyendo la historia de este, sus fundamentos teóricos y las demostraciones empíricas de su eficacia. Se confirma el compromiso de los padres para asistir al entrenamiento, ya que el programa es de naturaleza sumatoria; es decir, cada clase está construida sobre la información cubierta en clases anteriores. A los padres que asisten se les pide que se concentren en uno solo de sus hijos (niño-objetivo), e igualmente se les menciona la importancia de realizar los ejercicios solicitados en el libro de trabajo, los cuales sirven como apoyo personal inicial para la práctica de una crianza positiva y constante. En el presente estudio, para alentar la asistencia de los padres se implementó un sistema de incentivos acumulativo, utilizando boletos a los cuales se les ponía el nombre de su niño objetivo y en la última clase se rifó un objeto infantil.

La estructura de las clases sugiere que después de darles la bienvenida en cada sesión, se pida a los padres que contesten una prueba que viene incluida en su libro de trabajo. Esto se lleva a cabo antes de iniciar la presentación de los contenidos; al terminar la sesión igualmente se les pide que contesten la misma prueba. La intención de ese procedimiento es que los padres se den cuenta por sí mismos de la adquisición de conocimientos.

Cada clase, excepto la primera, se inicia facilitando una revisión del material cubierto en la clase anterior. A continuación se hace una introducción al tema, para luego revisar la tarea; esto tiene la doble intención, primero, de comprobar qué tan bien están aprendiendo los padres, y segundo, saber cómo están utilizando el PPE con su niño. El paso siguiente es presentar los nuevos conceptos que corresponden a cada clase. A continuación se describen los contenidos de las cuatro clases del programa:

- Primera Clase: El papel de los padres al educar al niño; explicación de las técnicas de paternidad ALTO-PIENSA.

- Segunda Clase: Desarrollo del niño; cómo establecer expectativas razonables; inclusión de la estrategia PREGUNTA. 
- Tercera Clase: Paternidad positiva; incorporación de la estrategia estrella ALTO, PIENSA, PREGUNTA y RESPONDE.

- Cuarta Clase: Cómo establecer límites; revisión de técnicas de disciplina y continuación de la técnica de RESPONDER; ilustración con numerosos ejemplos de su aplicación a la vida cotidiana.

Los padres se apoyan en escritos de crianza del mismo programa que facilitan la participación y la discusión, dejando espacios para que los demás participantes hagan preguntas y compartan sus experiencias. Cada clase concluye con un resumen del material revisado y se explica la tarea para la próxima sesión.

\section{Procedimiento}

En cada uno de los sitios se llevaron a cabo reuniones de orientación para explicar el proyecto y obtener el consentimiento firmado por los padres; se tuvo especial cuidado de los aspectos éticos, que incluyeron asegurar la confidencialidad de la información y el derecho de las familias a retirarse del estudio cuando lo desearan, de acuerdo con el Código Ético del Psicólogo (Sociedad Mexicana de Psicología, 2007). Los padres fueron invitados para asistir a la sesión de pre-evaluación (pretest), donde se aplicaron todos los cuestionarios. Posteriormente, 30 padres (i.e., mamás o papás) fueron asignados al azar al grupo experimental y se les ofreció el entrenamiento en crianza; los padres fueron capacitados en grupos de cuatro a seis personas e iniciaron esta preparación la semana siguiente a la preevaluación; al concluir el entrenamiento contestaron todos los auto-reportes nuevamente (fase de posttest). Los otros 30 padres formaron el grupo control, no recibieron ningún apoyo durante el estudio, y contestaron los auto-reportes en dos ocasiones (pretest y pre-evaluación II, diez semanas después de iniciado el estudio).

\section{RESULTADOS}

El objetivo de este estudio consistió en conocer si un entrenamiento en crianza para padres con niños de tres a cinco años de edad modificaría las prácticas disciplinarias severas, así como los efectos encontrados entre el corajeagresión y el estrés de los padres, el estrés en la interacción entre padres e hijos, y los problemas de conducta, el estrés de sus niños y esas prácticas. Para lograr el objetivo se planeó obtener correlaciones entre los puntajes de los factores considerados predictores potenciales de las prácticas disciplinarias y los de las propias prácticas, e igualmente realizar un análisis de regresión. Antes de efectuar esos análisis se procedió a corroborar el cumplimiento del supuesto de normalidad de las variables de este estudio. En la Tabla 1 se presentan las medias y desviaciones de las calificaciones de los padres en las seis variables del estudio. Se presentan también el sesgo y la kurtosis de cada variable; como se puede observar, los valores de todas las variables fueron cercanos a cero, indicando que las distribuciones fueron razonablemente simétricas y seguían la distribución normal. Además, la prueba de normalidad, KolmogorovSmirnov, no fue significativa $(p>.05)$ para ninguna variable. Con la confianza en que la distribución de cada variable se ajustaba al supuesto de normalidad, se obtuvieron las correlaciones Pearson entre prácticas disciplinarias y las conductas problema de los niños $(r=.47)$, el estrés de los padres $(r=.46)$, el del niño $(r=.52)$ y el de su interacción $(r=.42)$, y el coraje-agresión de los padres $(r=.41)$. Todas las correlaciones fueron significativas $(p<.001)$ indicando adecuada viabilidad para el análisis de regresión.

Tabla 1.

Datos descriptivos de la muestra total $(\mathrm{N}=60)$

\begin{tabular}{lcccc}
\hline \multicolumn{1}{c}{ Variable } & M & DT & Sesgo & Kurtosis \\
\hline Disciplina & 46.1 & 9.55 & 0.74 & 0.09 \\
Estrés-padres & 27.5 & 7.26 & 0.41 & -0.16 \\
Estrés-interacción & 22.0 & 7.20 & 0.66 & -0.02 \\
Estrés del niño & 28.2 & 7.62 & 0.21 & -0.80 \\
Conducta problema & 24.5 & 4.44 & 0.38 & 0.16 \\
Coraje/agresión & 9.23 & 3.93 & 0.21 & -0.23 \\
\hline
\end{tabular}

Se realizó un análisis de regresión jerárquica escalonado hacia adelante para la muestra total. Las prácticas disciplinarias fueron la variable dependiente, y las variables independientes fueron: las conductas problema y el estrés de los niños, el coraje-agresión y el estrés de los padres, y el estrés de la interacción padres-niños. En la Tabla 2 se observa que durante la fase de pre-evaluación, la primera variable en entrar a la ecuación como predictora de las prácticas disciplinarias fue el estrés en la interacción padresniños, y en dicha tabla se indica su efecto significativo; el coeficiente de determinación de esta variable $\left(R^{2}=.27, p<\right.$ $.001)$ muestra también que el $27 \%$ de la varianza explicada se debió al estrés en la interacción. En el segundo paso entró en la ecuación la conducta problema de los niños y en la Tabla 2 se observa que, aunque la contribución de esta variable por sí misma fue mucho menor $\left(D R^{2}=.07\right)$, también fue significativa; el coeficiente de determinación de esta variable y la anterior $\left(R^{2}=.35, p<.01\right)$ indica 
también que el $35 \%$ de la varianza explicada para prácticas disciplinarias se debió al estrés en la interacción y al comportamiento problema de los niños. En el tercer y último paso entró el coraje-agresión, pero su contribución individual a la varianza de las prácticas disciplinarias no fue significativa $(p>.05)$. La solución final fue, $R=.61$, $R^{2}=.37, F(3,56)=11.3, p<.001$.

Tabla 2.

Análisis jerárquico de la muestra global

\begin{tabular}{lcccc}
\hline \multicolumn{1}{c}{ Variables } & $\mathrm{B}$ & $\mathrm{EE}$ & $\beta$ & $\mathrm{DR} 2$ \\
\hline & \multicolumn{1}{c}{ Criterio: Disciplina } & & \\
\hline Paso 1 & & & & $.27^{* * *}$ \\
Estrés-interacción & .43 & .16 & $.32^{* *}$ & \\
Paso 2 & $.07^{* *}$ & & & \\
Conducta problema & .59 & .25 & $.27^{*}$ & \\
Paso 3 & .02 & & & \\
Coraje-agresión & .44 & .18 & .18 & \\
\hline
\end{tabular}

${ }^{*} p<.05 ;{ }^{* *} p<0.01 ;{ }^{* * *} p<.001$.

Una vez identificados los predictores de las prácticas disciplinarias, la muestra se dividió al azar en dos grupos; un grupo experimental (GE) con padres (20 mamás, 10 papás) que recibieron el entrenamiento en crianza, y uno control (GC) que no lo recibió (21 mamás, 9 papás). Diez semanas después, ambos grupos recibieron los mismos cuestionarios para que fueran contestados por ellos nuevamente. Para comprobar si todos los puntajes de las variables del GE se habían modificado por el entrenamiento, se planeó obtener MANOVA de medidas repetidas; sin embargo, debido a que las intervenciones afectan la distribución de los datos y, por ende, el supuesto de normalidad, se realizaron también pruebas no paramétricas. El MANOVA para las puntuaciones de las variables indicó efectos principales significativos $(F(5,25)=5.79, p<0.001)$; los análisis univariados confirmaron que el cambio en todas ellas fue significativo ( $p<.01$, por lo menos). Además, para comprobar si hubo cambios en los puntajes de disciplina, se realizó aparte una prueba $t$ de Student para muestras dependientes; esta prueba indicó que las puntuaciones de la fase de pre-evaluación a la de post-entrenamiento del GE habían disminuido de manera significativa $(t(29)=$ $-6.81, p<.001)$. Los análisis no paramétricos con la prueba Friedman-ANOVA de medidas repetidas (StatSoft, 2010) confirmaron los resultados obtenidos con pruebas paramétricas, ya que fueron significativos $(N=30, g l=1, p<$ .01 , por lo menos) para todas las variables; los valores del análisis se presentan después del nombre de cada variable: comportamiento problema (8.33), estrés parental (12.4), estrés del niño (9.14), estrés de la interacción (7.00), coraje/ agresión (11.6) y disciplina (22.5).

Para el GC, el MANOVA de medidas repetidas para las puntuaciones de las variables indicó que no había efectos significativos $(F(5,25)=1.52$, NS $)$. La prueba $t$ de Student para las puntuaciones de disciplina de la fase de pre-evaluación a la de pre-evaluación-II indicó que tampoco hubo cambios en esta variable $(t(29)=0.16$, NS). El análisis con la prueba Friedman-ANOVA confirmó que no hubo cambios para todas las variables $(N=30, g l=1)$; sus valores y probabilidades exactas se presentan después del nombre de cada variable: comportamiento problema $(1.00, p=.32)$, estrés parental $(2.28, p=.13)$, estrés del niño $(0.03, p=.85)$, coraje/agresión $(0.61, p=.43)$ y disciplina $(0.15, p=.69)$; no se reporta estrés de la interacción porque el análisis no fue posible debido a la ausencia de varianza, ya que los puntajes y sus desviaciones estándar en las dos evaluaciones fueron muy semejantes (pre: $M=20.9, D T$ = 6.7; pre-II: $M=21.1, D T=7.4$ ).

Además, se decidió reportar las estadísticas descriptivas de las puntuaciones obtenidas por los grupos GE y GC en los autoreportes contestados diez semanas después de la primera evaluación. Se decidió también realizar pruebas paramétricas fundamentadas en el hecho de que las violaciones menores del supuesto de normalidad no afectan los resultados (StatSoft, 1995; p. 1417), como se pudo constatar en los análisis anteriores. En la Tabla 3 se presentan los datos de las comparaciones con pruebas $t$ de Student para muestras independientes; estas indican que aunque todas las puntuaciones del GC eran mayores que las del GE, solo se encontraron diferencias altamente significativas para las prácticas disciplinarias y las conductas problema de los niños.

Tabla 3.

Comparación de las puntuaciones de los grupos, experimental $(\mathrm{n}=$ 30) y control $(n=30)$, diez semanas después

\begin{tabular}{l|c|c|c|c|c}
\hline \multicolumn{6}{c}{ Gr. Experimental Gr. Control } \\
\hline \multicolumn{1}{c|}{ Variables } & $\mathrm{M}$ & $\mathrm{DT}$ & $\mathrm{M}$ & $\mathrm{DT}$ & $\mathrm{t}($ g.1. 58) \\
\hline Disciplina & 35.3 & 5.08 & 46.2 & 11.1 & $-4.86^{*}$ \\
Estrés de los padres & 22.5 & 7.61 & 25.1 & 6.30 & -1.42 \\
Estrés - interacción & 19.6 & 7.55 & 21.1 & 7.38 & -0.79 \\
Estrés del niño & 23.7 & 7.14 & 27.2 & 7.44 & -1.87 \\
Conducta problema & 20.2 & 3.51 & 23.7 & 4.09 & $-3.55^{*}$ \\
Coraje/agresión & 7.16 & 4.54 & 8.50 & 3.89 & -1.21 \\
\hline
\end{tabular}

${ }^{*} p<.001$. 
Finalmente, para lograr el objetivo se realizaron dos análisis de regresión. En la Tabla 4 se muestran los resultados de los análisis de regresión jerárquica escalonados hacia adelante para cada grupo. Los análisis indicaron que para el GE la única variable predictora en la ecuación fue el coraje-agresión y se indica en esa Tabla su efecto significativo que, por supuesto, es semejante a la solución final $\left(R=.49, R^{2}=.24, F(1,28)=9.23, p<.01\right)$; el coeficiente de determinación de esta variable indica que el $24 \%$ de la varianza explicada se debía al coraje-agresión de los padres.

Tabla 4.

Análisis jerárquico del grupo experimental y del grupo control

\begin{tabular}{lcccc}
\hline \multicolumn{1}{c}{ Variables } & B & EE & $\beta$ & DR2 \\
\hline Grupo Experimental & & & & \\
\hline Criterio: Disciplina & & & & \\
\hline Paso 1 & & & & $.24^{* * *}$ \\
\hline Coraje-agresión & .55 & .18 & $.49^{* * *}$ & \\
\hline Grupo Control & & & & \\
\hline Criterio: Disciplina & & & & \\
\hline Paso 1 & & & & $.43^{* * *}$ \\
\hline Estrés-interacción & .52 & .26 & $.34^{*}$ \\
\hline Paso 2 & & & & $.10^{*}$ \\
\hline Coraje-agresión & .86 & .40 & $.30^{*}$ \\
\hline Paso 3 & & & \\
\hline Estrés de los padres & .51 & .31 & .28 \\
\hline
\end{tabular}

$" p<.05 ; " * p<.01 ; * " * x<.001$.

En contraste, para el GC se observa en la Tabla 4 que la primera variable en entrar a la ecuación como predictora de las prácticas disciplinarias fue el estrés en la interacción padres-niños y se indica en esa Tabla su efecto significativo; el coeficiente de determinación de esta variable $\left(R^{2}\right.$ $=.43, p<.001)$ indica también que el $43 \%$ de la varianza explicada se debió al estrés en la interacción. En el segundo paso entró en la ecuación el coraje-agresión de los padres y en la Tabla se observa que, aunque la contribución de esta variable por sí misma fue menor, también fue significativa; el coeficiente de determinación $\left(R^{2}=.53, p<.05\right)$ indica también que el $53 \%$ de la varianza explicada para prácticas disciplinarias se debió a estas dos variables. En el tercer y último paso entró el estrés de los padres, pero su contribución individual a la varianza de las prácticas disciplinarias no fue significativa $(p>.05)$. La solución final con estas variables fue, $R=.76, R^{2}=.58, F(3,26)$ $=12.0, p<.001$.

\section{DISCUSIÓN}

Para poder apreciar mejor el significado de los resultados se resumen aquí las características de la muestra estudiada; aunque esta fue relativamente pequeña en tamaño, fue lo suficientemente diversa en ingresos y escolaridad; dos terceras partes eran mamás y el resto papás, en su mayoría casados, adultos-jóvenes y con empleo. Es conveniente señalar también que las puntuaciones de esta muestra durante la fase de pre-evaluación fueron semejantes a las reportadas en otros estudios con niños pequeños (e.j., Solís-Cámara et al., 2004).

En el presente estudio, durante la fase de pre-evaluación, se encontraron relaciones altamente significativas entre las prácticas disciplinarias de los padres y todos los posibles determinantes, pero las correlaciones más fuertes se encontraron para el estrés paterno y el del niño, y para las conductas problema. Las relaciones entre la disciplina severa, o castigo, y el estrés y las conductas problema están muy bien documentadas en la literatura especializada (Anthony et al., 2005; Conger et al., 2003; Guajardo et al., 2009; McBride et al., 2002; Pinderhughes, Dodge, Bates, Pettit \& Zelli, 2000; Solís-Cámara et al., 2004), por lo que no fue sorprendente confirmarlas, pero ofrece confianza en los resultados obtenidos.

El análisis de regresión con la muestra total mostró que los determinantes potenciales de las prácticas disciplinarias no tenían la misma importancia, siendo el estrés en la interacción y las conductas problema, los más fuertemente relacionados con la disciplina que se apoya en golpes y gritos. El coraje-agresión entró también en el modelo, pero su contribución fue secundaria a los otros determinantes. Estos resultados indican que a mayor estrés en la relación padres-niños y a mayor número de conductas problema, las prácticas disciplinarias son más severas.

Previo a la discusión de los análisis de regresión por grupos, es importante señalar que en la comparación de las puntuaciones del grupo experimental, de la fase de pre-evaluación a la de post-entrenamiento, no solo se modificaron significativamente las prácticas disciplinarias sino todas las variables incluidas. En contraste, los puntajes de la pre-evaluación a la segunda evaluación en el grupo control no se modificaron de manera significativa, como sería ideal esperar.

Los análisis de regresión realizados diez semanas después de la primera evaluación indicaron que en el grupo experimental los determinantes significativos de las prácticas disciplinarias severas perdían su peso predictivo (i.e., estrés en la interacción y las conductas problema), pero el coraje-agresión tomaba relevancia como determinante de 
la disciplina de los padres. Estos resultados indican que la intervención realizada modificó todos los determinantes relacionados con la disciplina y también las propias prácticas disciplinarias severas, lo cual no es sencillo de lograr; por ejemplo, un estudio reciente reportó que no se encontró reducción del estrés de los padres después de su entrenamiento (Fajardo-Vargas \& Hernández-Guzmán, 2008). De acuerdo con Aneshensel (1996), el estrés en sus diversas modalidades puede considerarse de consecuencias secundarias, en el sentido de que el estrés experimentado por los padres tiene consecuencias indirectas vía la alteración de prácticas parentales eficientes, y de ahí su efecto negativo en los niños. En cuanto al análisis de regresión con el grupo control, este indicó que su comportamiento continuaba siendo semejante al de la muestra total. Esto es correcto particularmente en cuanto al peso de la relación entre las prácticas disciplinarias más severas y el estrés en la interacción padres-niños; sin embargo, es interesante notar en este análisis que las conductas problema de los niños no entraron en el modelo, y el coraje-agresión resaltó más.

Por otra parte, las comparaciones entre las puntuaciones post-entrenamiento del grupo experimental y las puntuaciones del grupo control en la fase de pre-evaluación-II indicaron diferencias significativas solo en la disciplina y en las conductas problema entre ambos grupos. Este resultado se puede explicar en el sentido del decremento simultáneo y muy importante de las prácticas disciplinarias severas y las conductas problema en el grupo experimental. Como vimos antes en este estudio, las evidencias indican una estrecha relación entre estas variables (Brunk \& Henggeler, 1984; Goldberg et al., 2002; Pike et al., 1996). Patterson (2002) ha señalado que los castigos severos favorecen una escalada de comportamientos disruptivos en los niños por medio del modelamiento y el reforzamiento negativo, y con base en esas evidencias, Patterson y Fisher (2002) "...han enfatizado la necesidad de enseñar a los padres cómo proveer un conjunto efectivo de castigos no físicos para el comportamiento inadecuado del niño.” (p. 73). En consecuencia, un entrenamiento como el aquí utilizado, que facilita el uso adecuado de técnicas cognitivo-conductuales pudo modificar positivamente las relaciones negativas entre los padres y el niño.

En la muestra total se observó que, a mayor corajeagresión, mayor severidad en las prácticas disciplinarias. Aunque el peso de esta relación fue secundario al del estrés en la interacción y los problemas de conducta en el análisis de regresión, una vez descartadas estas últimas relaciones por el entrenamiento a padres, la importancia del coraje-agresión fue más clara. Para reforzar aún más esta idea, es importante notar que el coraje-agresión de los padres del grupo control tuvo mayor peso predictivo para las prácticas disciplinarias severas diez semanas después de iniciado el estudio. Evidencia reciente indica que el coraje y la agresión materna tiene influencia directa, no mediada por otros factores, en los problemas de conducta de los niños (Fava, 2013).

El hecho de que el entrenamiento a los padres no haya tenido éxito para modificar la relación entre el corajeagresión y las prácticas disciplinarias es congruente con el argumento de Erath y colegas (Erath, Bierman \& Conduct Problems Prevention Research Group, 2006) de que los programas para padres pueden no tener el alcance suficiente para proteger a los niños de la agresión parental que puede resultar de otros factores, por ejemplo, del conflicto entre los padres; lo que puede requerir de intervenciones más específicas dirigidas al manejo del coraje.

Hace 30 años, Belsky (1984) señaló que la crianza es multideterminada por fuerzas que la debilitan o la fortalecen, y que considerar la sola contribución de alguna de estas fuerzas no era suficiente para comprender por qué los padres educan a sus hijos como lo hacen, sino la acumulación de diversas fuerzas estresantes o de apoyo; este concepto sigue vigente en la actualidad (Baumrind et al., 2002). El presente estudio apoya la importancia de múltiples determinantes de las prácticas disciplinarias severas. Aunque otros estudios indican que es importante tener presente que los hallazgos que relacionan los "determinantes" con las prácticas de crianza sólo indican procesos causales potenciales (Losoya, Callor, Rowe \& Goldsmith, 1997; Spinath \& O’Connor, 2003), para tener un mayor conocimiento de los efectos de las prácticas disciplinarias en los niños, probablemente se requiera realizar observaciones de estos, lo cual puede ser una limitación de este y de otros estudios.

Finalmente, las principales aportaciones de este estudio fueron identificar predictores potenciales de las prácticas disciplinarias severas y pruebas empíricas de su modificabilidad, que confirman los resultados de otros estudios (Nicholson et al., 2002; Solís-Cámara et al., 2000). El entrenamiento realizado facilitó el uso de técnicas cognitivo-conductuales, lo cual pudo modificar positivamente las relaciones negativas entre los padres y el niño. El conocimiento de factores predisponentes y su modificabilidad puede ser útil para la práctica de la psicología, en particular la dirigida a las familias y a sus niños, y sugiere que programas para padres, como el ofrecido aquí (Solís-Cámara et al., 2001), pueden ser aplicados en los consultorios y/o agencias de apoyo social de la familia. 


\section{REFERENCIAS}

Abidin, R. R. (1995). Parenting Stress Index (3ra. ed.). Odessa, FL.: Psychological Assessment Resources.

Aneshensel, C. S. (1996). Consequences of Psychosocial Stress. The Universe of Stress Outcomes. En H. B. Kaplan (Ed.), Psychosocial Stress. Perspective on Structure, Theory, LifeCourse, and Methods (pp. 111-136). San Diego, CA: Academic Press.

Anthony, L., Anthony, B., Glanville, D. N., Naiman, D. Q., Waanders, C. \& Shaffer, S. (2005). The Relationship between Parenting Stress, Parenting Behaviour and Preschoolers' Social Competence and Behaviour Problems in the Classroom. Infant and Child Development, 14(2), 133-154. doi:1 0.1002/icd.385.

Atkins, Marc S., Frazier, J. A. A. \&Talbott, E. (2003). SchoolBased Mental Health Services in Urban Communities. En M. D. Weist, S. W. Evans \& N. A. Lever (Eds.), Handbook of school mental health: Advancing Practice and Research (pp. 275-285). New York: Kluwer Academic/Plenum Publishers.

Baumrind, D., Larzelere, R. E. \& Cowan, P. A. (2002). Ordinary Physical Punishment: Is it Harmful? Comment on Gershoff (2002). Psychological Bulletin, 128(4), 580-589. doi.org/10. 1037/0033-2909.128.4.602.

Berzenski, S. R. \& Yates, T. M. (2013). Preschoolers' Emotion Knowledge and the Differential Effects of Harsh Punishment. Journal of Family Psychology, 27(3), 463-472. doi. org/10.1037/a0032910.

Belsky, J. (1984). The Determinants of Parenting: A process Model. Child Development, 55(1), 83-96. doi.org/10.2307/1129836.

Belsky, J. (1990). Parental and Nonparental Child Care Children's Socioemotional Development: A Decade in Review. Journal of Marriage and the Family, 52, 885-903. doi. org/10.2307/353308.

Belsky, J. \& Barends, N. (2002). Personality and Parenting. En: M. H. Bornstein (Ed.), Handbook of parenting, Vol 3. Being and becoming a parent (2da ed., pp. 415-438). Mahwah, NJ: Lawrence Erlbaum Associates. doi.org/10.4324/9781410612151.

Belsky, J. \& Jaffee, S. R. (2006).The Multiple Determinants of Parenting. En: D.

Cicchetti\& D. Cohen (Eds.), Developmental psychopathology, Vol 3, Risk, disorder, and adaptation (2da ed., pp. 38-85). New York, NY: Wiley.

Brunk, M. A. \& Henggeler, S. W. (1984).Child Influences on Adult Controls: An Experimental Investigation. Developmental Psychology, 20(6), 1074-1081. doi.org/10.1037/ 0012-1649.20.6.1074.

Bugental, D. B. \& Happaney, K. (2002). Parental attributions. En: M. H. Bornstein (Ed.), Handbook of parenting, Vol 3. Being and becoming a parent (2da ed., pp. 509-535). Mahwah, NJ: Lawrence Erlbaum Associates.

Clark, L. A., Kochanska, G. \& Ready, R. (2000). Mothers' Personality and its Interaction with Child Temperament as Predictors of Parenting Behavior. Journal of Personality and
Social Psychology, 79(2), 274-285. doi.org/10.1037//00223514.79.2.274.

Collins, W. A., Maccoby, E. E., Steinberg, L., Hetherington, E. M. \& Bornstein, M. H. (2000). Contemporary Research onParenting. The Case for Nature and Nurture. American Psychologist, 55(2), 218-232. doi.org/10.1037//0003-066x.55.2.218.

Conger, R. D., Neppl, T., Kim, K. J. \& Scaramella, L. (2003). Angry and Aggressive Behavior across Three Generations: A Prospective, Longitudinal Study of Parents and Children. Journal of Abnormal Child Psychology, 31(2), 143-160. doi. org/10.1023/a:1022570107457.

Dallaire, D. H. \& Weinraub, M. (2005).The Stability of Parenting Behaviors over the First 6 Years of Life. Early Childhood Research Quarterly, 20, 201-219. doi.org/10.1016/j. ecresq.2005.04.008.

Dishion, T. J. \& Patterson, G. R. (1992). Age Effects in Parent Training Outcome. Behavior Therapy, 23, 719-729. doi. org/10.1016/s0005-7894(05)80231-X.

Erath, S. A., Bierman, K. L. \& Conduct Problems Prevention Research Group (2006). Aggressive Marital Conflict, Maternal Harsh Punishment, and Child Aggressive-Disruptive Behavior: Evidence for Direct and Mediated Relations. Journal of Family Psychology, 20(2), 217-226. doi.org/10.1037/08933200.20.2.217.

Fajardo-Vargas, V. \& Hernández-Guzmán, L. (2008). Tratamiento cognitivo-conductual de la conducta agresiva infantil. Revista Mexicana de Análisis de la Conducta, 34(2), 371-389. doi. org/10.5514/rmac.v34.i2.16216.

Fava, J. L. (Agosto, 2013). Maternal Anger and Harsh Discipline in Relation to Child Emotion, Dysregulation and Behavior Problems. Ponencia presentada en la Convención Anual de la American Psychological Association, Honolulu, Hawaii. doi.org/10.1037/e591562013-001.

Gershoff, E. T. (2002). Parental Corporal Punishment and Associated Child Behaviors and Experiences: AMeta-Analytic and Theoretical Review. Psychological Bulletin, 128, 539-579. doi.org/10.1037/0033-2909.128.4.539.

Grych, J. H. (2002). Marital Relationships and Parenting. En: M. H. Bornstein (Ed.), Handbook of parenting, Vol 4. Social conditions and applied parenting (2da ed., pp. 203-225). Mahwah, NJ: Lawrence Erlbaum Associates.

Goldberg, W. A., Clarke-Stewart, K. A., Rice, J. A. \& Dellis, E. (2002). Emotional Energy as an Explanatory Construct for Fathers' Engagement with their Infants. Parenting: Science and Practice, 2(4), 379-408. doi.org/10.1207/ s15327922par0204_03.

Guajardo, N. R., Snyder, G. \& Petersen, R. (2009). Relationships among Parenting Practices, Parental Stress, Child Behaviour, and Children's Social-Cognitive Development. Infant and Child Development, 18, 37-60. doi.org/10.1002/icd.578.

Hansen, K. \& Jones, E. M. (2010). Age 5 Cognitive Development in England. Child Indicators Research, 3, 105-126. doi. org/10.1007/s12187-009-9055-5. 
Hill, N. E. (2006). Disentangling Ethnicity, Socioeconomic Status and Parenting: Interactions, Influences and Meaning. Vulnerable Children and Youth Studies, 1(1), 114-124. doi. org/10.1080/17450120600659069.

Lindahl, K. M. \& Malik, N. M. (1999). Observations of Marital Conflict and Power: Relations with Parenting in the Triad. Journal of Marriage and the Family, 61(2), 320-330. doi. org/10.2307/353751.

Lochman, J. E. \& Wells, K. C., (2003). Effectiveness Study of Coping Power and Classroom Intervention with Aggressive Children: Outcomes At A One-Year Follow Up. Behavior Therapy, 34, 493-515. doi.org/10.1016/s0005-7894(03)80032-1.

Losoya, S. H., Callor, S., Rowe, D. C. \& Goldsmith, H. H. (1997). Origins of Familial Similarity in Parenting: A Study of Twins and Adoptive Siblings. Developmental Psychology, 33(6), 1012-1023. doi.org/10.1037//0012-1649.33.6.1012.

Lynch, S. K., Turkheimer, E., D’Onofrio, B. M., Mendle, J., Emery, R. E. \& Slutske, W. S. (2006). A Genetically Informed Study of the Association between Harsh Punishment and Offspring Behavioral Problems. Journal of Family Psychology, 20(2), 190-198. doi.org/10.1037/0893-3200.20.2.190.

Maiuro, R. D., Vitaliano, P. P. \& Cahn, T. S. (1987). A Brief Measure for the Assessment of Anger and Aggression. Journal of Interpersonal Violence, 2, 166-178. doi. org/10.1177/088626087002002003.

Margolin, G., Oliver, P.H. \& Medina, A. M. (2001). Conceptual Issues in Understanding the Relation between Interparental Conflict And Child Adjustment: Integrating Developmental Psychopathology and Risk/Resilience Perspectives. En: J. H. Grych \& F. D. Fincham (Eds.), Interparental Conflict and Child Development: Theory, Research, and Applications (pp. 9-38). New York, NY: Cambridge University Press. doi. org/10.1017/cbo9780511527838.003.

McBride, B. A., Schoppe, S. J. \& Rane, T. R. (2002). Child Characteristics, Parenting Stress, and Parental Involvement: Fathers versus Mothers. Journal of Marriage and the Family, 64(4), 998-1011. doi.org/10.1111/j.1741-3737.2002.00998.x.

NICHD Early Child Care Research Network. (1999). Chronicity of Maternal Depressive Symptoms, Maternal Sensitivity, and Child Functioning at 36 Months. Developmental Psychology, 35(5), 1297-1310.

Nicholson, B., Anderson, M., Fox, R. \& Brenner, V. (2002). One Family at a Time: A Prevention Program for At-Risk Parents. Journal of Counseling and Development, 80, 362-372. doi. org/10.1002/j.1556-6678.2002.tb00201.x

Patterson, G. R. (2002).Etiology and Treatment of Child and Adolescent Antisocial Behavior.The Behavior Analyst Today, 3, 133-144. doi.org/10.1037/h0099971.

Patterson, G. R. \& Fisher, P. A. (2002).Recent Developments in Our Understanding of Parenting: Bidirectional Effects, Causal Models, and The Search For Parsimony. En: M. H. Bornstein (Ed.), Handbook of parenting Vol. 5: Practical issues in parenting (pp. 59-88). Mahwah, NJ: LEA.
Pike, A., McGuire, S., Hetherington, E. M., Reiss, D. \& Plomin, R. (1996). Family Environment and Adolescent Depressive Symptoms and Antisocial Behavior: A Multivariate Genetic Analysis. Developmental Psychology, 32(4), 590-603. doi. org/10.1037/0012-1649.32.4.590.

Pinderhughes, E. E., Dodge, K. A., Bates, J. E., Pettit, G. S. \& Zelli, A. (2000). Discipline Responses, Influences of Parents' Socioeconomic Status, Ethnicity, Beliefs about Parenting, Stress, and Cognitive-Emotional Processes. Journal of Family Psychology, 14(3), 380-400. doi.org/10. 1037//08933200.14.3.380.

Ragozin, A. S., Basham, R. B., Crnic, K. A., Greenberg, M. T. \& Robinson, N. M. (1982). Effects of Maternal Age on Parenting Role. Developmental Psychology, 18(4), 627-634. doi.org/10.1037/0012-1649.18.4.627.

Reid, M. J., Webster-Stratton, C. \& Beauchaine, T. P. (2001). Parent Training inHead Start: A Comparison of Program Response among African American, Asian American, Caucasian, and Hispanic mothers. Prevention Science, 2(4), 209-227.

Richman, M. \& Graham, P. J. (1971). A Behavioral Screening Questionnaire for Use with Three Year Old Children. Journal of Child Psychology and Psychiatry, 12, 5-33. doi. org/10.1111/j.1469-7610.1971.tb01047.

Richman, M., Stevenson, J. E. \& Graham, P. J. (1975). Prevalence of Behavior Problems in 3-Year-Old Children: An Epidemiological Study in a London Borough. Journal of Child Psychology and Psychiatry, 16, 277-287. doi. org/10.1111/j.1469-7610.1975.tb00362.x.

Sociedad Mexicana de Psicología (2007).Código Ético del Psicólogo. México: Editorial Trillas.

Solís-Cámara, R. P. (2007). Manual de la Escala de Comportamientos de Madres y Padres con Niños Pequeños. Bogotá, Colombia: www.librosdepsicologia.com

Solís-Cámara, R. P., Covarrubias S., P., Díaz R., M. \& Rivera A., B. (2004). Efectos multidimensionales de un programa de crianza en la interacción recíproca de padres y sus niños con problemas de comportamiento. Psicología Conductual, 12(2), 197-214.

Solís-Cámara, R. P. \& Díaz-Romero, M. (2002). Efectos de un programa de crianza para mamás y papás de niños pequeños: La importancia del nivel educativo de los padres. Revista Latinoamericana de Psicología, 34, 203-215.

Solís-Cámara, R. P., Díaz-Romero, M., Del Carpio, P., Esquivel, E., Acosta, I. \& De Jesús, A. (2007). La contribución del bienestar subjetivo, las expectativas y la crianza maternas en los logros escolares de sus niños y en la valoración de la participación de los padres. Acta Colombiana de Psicología, 10, 2, 71-82.

Solís-Cámara, R. P., Díaz, M., Fox, R. A. \& Fox, T. (2001). Paternidad Estrella: Guía de líderes-extensa. Guadalajara, México: Paternidad Estrella, Inc.

Solís-Cámara, R. P., Díaz, M., Fox, R. A. \& Fox, T. (2002). Libro de Trabajo: Paternidad Estrella. Un enfoque positivo y 
práctico para la paternidad eficiente (3 a 5 años) (2da ed.). Guadalajara, México: Paternidad Estrella, Inc.

Solís-Cámara, R. P., Díaz-Romero, M., Medina, Y., Barranco, L., Montejano, H. \& Tiscareño, A. (2002). Estructura Factorial y propiedades de la Escala de Comportamientos para Madres y Padres de Niños Pequeños (ECMP). Psicothema, 14, 637-642.

Solís-Cámara, R. P. \& Fox R. A. (2007). Escala de comportamientos de madres y padres con niños pequeños. Instrumento, perfiles y plantillas, para computadora. Bogotá, Colombia: www.psicomeditores.com

Solís-Camara, R. P., Fox, R. A. \& Nicholson, B. C. (2000). Parenting Young Children: Comparison of a Psychoeducational Program in Mexico and the United States. Early
Child Development and Care, 163, 115-124. doi: 10.1080/ 0300443001630108.

Spinath, F. M. \& O’Connor, T. G. (2003). A Behavioral Genetic Study of the Overlap Between Personality and Parenting. Journal of Personality, 71(5), 785-808. doi.org/10.1111/14676494.7105004.

StatSoft (1995). Statistica Volume I: General Conventions \& Statistics I. StatSoft Inc.: Tulsa, OK, USA.

StatSoft (2010). Statistica 9.1. StatSoft Inc.: Tulsa, OK, USA.

Weymouth, L. A. (2010). Act Against Violence: A Multi-Site Evaluation of the Parents Raising Safe Kids Program. Tesis de maestría no publicada, California State University, Humboldt Campus, CA, EE.UU. 